\title{
Where's the Schema? A Taxonomy of Patterns for Software Exchange
}

\author{
Dean Jin James R. Cordy Thomas R. Dean \\ Queen's University, Kingston, Canada \\ \{jin, cordy\}@cs.queensu.ca, thomas.dean@ece.queensu.ca
}

\begin{abstract}
Program comprehension tools extract, organize and analyze information about the design and implementation of software systems. Before tools can exchange information, they must share, at some level, the organization for the data exchanged. That is, they must share a schema. In this paper we examine the various ways in which schemas are represented and used in tools. Schema use is classified according to how and where a schema is defined, leading to the identification of four patterns of exchange. We examine these exchange patterns and discuss how each has been used in existing tool integration technologies. An evaluation of each exchange pattern against the requirements for a standard exchange format reveal the pattern of schema use that is most suitable for integrating tools.
\end{abstract}

\section{Introduction}

Program comprehension tools extract, organize and analyze information about the design and implementation of existing software systems. Efforts towards integrating program comprehension tools have been severely hindered by the lack of a consistent model for the structural makeup of software representations. The development of a Standard Exchange Format (SEF) for software is seen as the desired solution to this problem [31, 24, 12].

Schemas that accommodate the representational needs of various program comprehension tools are an essential part of an SEF. In this paper, we examine the various ways in which schemas are represented and used in tools, and classify them according to two distinguishing characteristics. Based on the use of schemas, four different exchange patterns are distinguishable. We examine each of these exchange patterns and discuss how each has been used in existing program comprehension tool integration technologies. We also examine how each of the exchange patterns satisfy the SEF requirements suggested by St-Denis et al. [32]. This leads us to propose which of the exchange pat- terns is best suited for use in software exchange formats. As always, any evaluation of the properties of real software systems is somewhat subjective.

\section{Integration Technologies}

In this paper we restrict our discussion of integration technologies to those that have been put to use for representing software in the context of program comprehension. In particular we refer to the following:

Ada Semantic Interface Specification (ASIS) [1] An ISO ratified [17] open source API written in Ada95 for accessing information from the Ada95 Compilation Environment.

Annotated Terms (ATerms) [36, 35] An exchange format and an API that represents data produced by parsers, structural editors, compilers and other components in software reengineering tools.

InterMediate Language (IML) [7] A portable intermediate representation developed by Project Bauhaus [2].

Resource Graph (RG) [7] An exchange format for medium to high abstractions of source code developed by Project Bauhaus.

Common Object-based Re-engineering Unified Model (CORUM) [38] An API-based environment for integrating software reengineering tools that work at the source code level.

CORUM II [19] A proposal for enhancing CORUM to provide advanced functionality for analysis at the architectural level of abstraction.

Datrix-TA [21] A format for exchanging Datrix-formatted abstract semantic graphs (ASGs) [20] among the different tools that make up the Datrix system. 
FAMOOS Information Exchange Model (FAMIX) [33, 8] A portable intermediate representation for objectoriented source code.

Graph eXchange format (GraX) [9, 10] A format for exchanging software representations as TGraphs [11].

Graph Exchange Language (GXL) $[15,16]$ A flexible format for exchanging software representations at all levels of abstraction.

PROgramming with Graph Rewriting Systems (PROGRES) [29, 30] The format used in the PROGRES environment, an integrated set of freeware tools that help developers create, analyze, compile and debug specifications for graph rewriting systems.

Rigi Standard Format (RSF) A format for exchanging high-level representations of software systems used by the Rigi [26] tool.

Tuple-Attribute (TA) [13] A flexible tuple-based language for expressing and exchanging software representations.

$T A++$ [22] A modified version of $T A$ used for representing and manipulating software representations among components that make up the TkSee [34] tool.

These integration technologies can be distinguished using characteristics such as [18]:

- The data structure used to represent software.

- The level of abstraction supported.

- The encoding method used.

- The mechanism used to transfer representations among program comprehension tools.

- The ability to change the structure and interpretation of data represented.

All of these integration technologies share a common purpose: to enable the portability of structured information among different systems. Within the program comprehension domain, representations of software are the structured information whose exchange is enabled. Almost all representations are constructed using some variant of an entityrelationship (E-R) model [5]. E-R models provide a clean separation between the information that defines the allowable characteristics of a model and the data that is represented in the model. The former is known as schema and the latter as instance.

In terms of integration, this schema-instance severance provides a significant advantage. Negotiation of exchange among tools requires communal knowledge of the structure of information to be passed. For a given software model, this information is readily available in the schema. So the schema plays a pivotal role in the exchange process.

\section{Schema Classification}

Ultimately, the way a schema is used dictates how a tool will negotiate exchange with other tools. We classify the use of schemas in two dimensions: schema definition and schema locality.

\subsection{Schema Definition}

Schema definition characterizes how the schema is defined. Within this dimension two classes of schema definition are identified:

Implicit. The structure of the representation is not explicitly documented, but rather is implied by the data itself or its use.

Explicit. The structure of the representation is explicitly documented, either through a specification or some other means.

\subsection{Schema Locality}

Schema locality distinguishes where the schema is defined. Within this dimension two classes of schema locality are identified:

Internal. The schema is an integral part of the tool. As a consequence, the schema is not required to participcate in the exchange.

External. The schema definition is external to the tool. Because of this detachment, the schema is a participant in the exchange that occurs between tools.

The distinction between internal and external schema locality is particularly important because external schemas represent a contract between tools independent of their own use. External schemas also provide the opportunity for tools to automatically adapt to a given schema.

\section{Exchange Patterns}

According to the schema classifications outlined above, four different types of exchange can be negotiated among program comprehension tools. We refer to these types of exchange as exchange patterns. We will now characterize the exchange patterns and provide examples for each. Figures 1 to 4 show the relationship between the tool, the schema and the representation of the software. Each figure consists of the following components: 
- A tool T.

- A schema S.

- I and I' respectively representing the state of a software representation instance before and after it is processed by tool $\mathrm{T}$.

In Figure 1 an exchange that uses an implicit-internal schema is shown. The schema is embedded in the code, so it is found in many locations within the tool. Tools that are built to make use of an API to exchange software representations fall into this category. APIs essentially have a fixed schema, so the tools that use them are constructed according to an implicit yet predetermined concept of the software representation being exchanged.

Exchange that uses an explicit-internal schema is shown in Figure 2. Although the schema remains an integral part of the tool, it is provided as a specification so the schema is shown in a single location. Tools constructed within the PROGRES environment make use of schemas in this fashion. The tool developer first provides a schema in the form of a specification that outlines the graph-based structure of data to be represented and the operations that can be performed on them. Transactions that work with graph instances provide the functionality for the tool being constructed.

In Figures 3 (a) and (b) we see two exchange patterns that use an explicit-external schema. The tool shown in Figure 3 (a) receives the schema first followed by the instance data. The integration technologies GraX and GXL work in this fashion. Schema and instance data are stored separately and all data instances provide a link to the file where the schema is stored. In Figure 3 (b) the schema and the instance data are received simultaneously. The integration technology $T A$ works this way. The schema information stored in the scheme tuple and scheme attribute sections are exchanged along with instance data stored in the fact tuple and fact attribute sections of the same file.

Exchange that uses an implicit-external schema is shown in Figure 4. In this case, the schema does not exist (so it is shown in a box with a dashed border) yet it does dictate the structural semantics of the information exchanged. The integration technology $R S F$ is an example of an exchange format that works this way. The use of a tuple notation is a syntactic requirement. The implicit schema for the information exchanged is an unconstrained E-R model. Tools such as Rigi [26] and Holt's Grok [14] accept E-R models in RSF. These tools have been pre-configured to handle constraintfree E-R instance data, so there is no need for a schema. In essence, they discover the schema from the instance data.

The exchange patterns used for each of the program comprehension tool integration technologies are shown in Table 1.

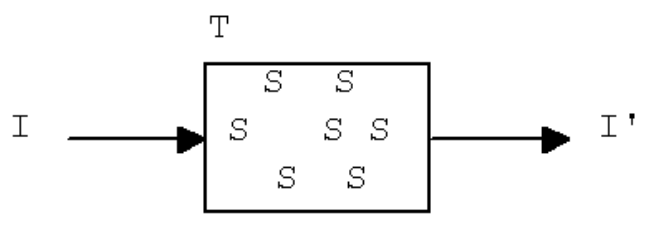

Figure 1. Exchange Using An Implicit-Internal Schema

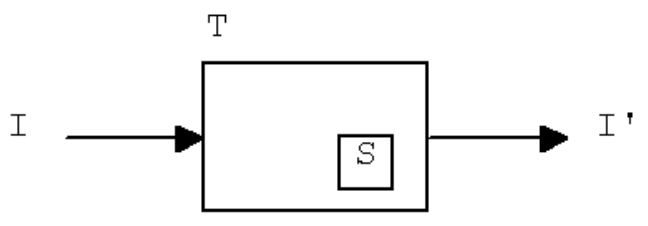

Figure 2. Exchange Using An Explicit-Internal Schema

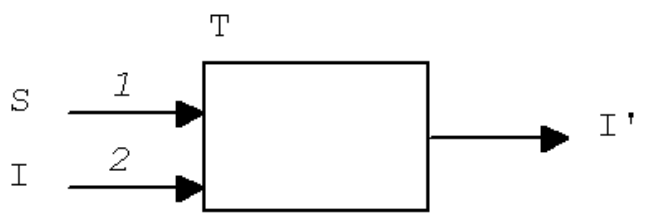

Figure 3. (a) Exchange Using An ExplicitExternal Schema with Consecutive Receipt of Schema and Instance

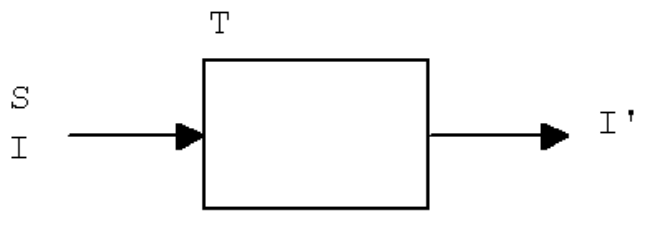

Figure 3. (b) Exchange Using An ExplicitExternal Schema with Simultaneous Receipt of Schema and Instance

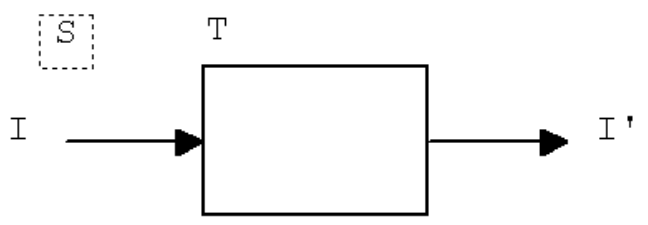

Figure 4. Exchange Using An ImplicitExternal Schema 


\begin{tabular}{|c|c|c|c|}
\hline & \multicolumn{2}{|c|}{ Schema Definition } \\
\hline & & Implicit & Explicit \\
\hline \multirow{2}{*}{$\begin{array}{l}\text { Schema } \\
\text { Locality }\end{array}$} & Internal & $\begin{array}{l}\text { ASIS, CORUM, } \\
\text { CORUM II, IML }\end{array}$ & $\begin{array}{c}\text { ATerms, } \\
\text { PROGRES }\end{array}$ \\
\hline & External & RSF & $\begin{array}{c}\text { Datrix-TA }{ }^{1}, \text { FAMIX }^{2}, \text { GraX }^{2} \\
\text { GXL }^{2}, \text { RG }^{1}, \text { TA }^{1}, \mathrm{TA}_{++}{ }^{1}\end{array}$ \\
\hline
\end{tabular}

Table 1. Exchange Pattern Used By Various Program Comprehension Tool Integration Technologies

\section{Advantages and Disadvantages}

As we mentioned, schema definition is a characterization of how the schema is defined, while schema locality relates to where the schema definition takes place. We now consider the advantages and disadvantages of each of schema classification in relation to its use in exchange.

\subsection{Implicit Schema Definitions}

The main advantage of an implicit schema definition is that it provides good performance. There is no need to carry out any additional processing or manage specifications for the representation being used. When the implicit definition is located internally, the representation is close at hand, being built into the code for the tool. Even when the implicit definition is external, the tool knows the structure of the information being exchanged so the opportunity to handle it appropriately is provided. This typically translates into the ability to processes large quantities of information in a fast and efficient manner. This is especially beneficial for program comprehension tools that work with source whose magnitude is measured in millions of lines of code.

A number of disadvantages offset the performance advantages of an implicit schema definition. Because the definition is static by nature, the representation is not extensible. This is a major problem for tools that are built around a particular information model. In such a situation, making changes to the representation involves a wholesale revision of code. Documentation is also a problem when the schema definition is implicit. A separate document outlining the structure and semantics of the representation is a necessity. Maintaining this documentation is time consuming and keeping it in sync with tool or exchange format changes is especially challenging.

A third problem with implicit schema definitions relates to the manner in which tools typically accept input. It is often useful to verify the integrity of information being exchanged. This usually involves a check to ensure that the input is well-formed. When the schema definition is implicit, such a test is difficult to implement and maintain. The

\footnotetext{
${ }^{1}$ Employs simultaneous receipt of schema and instance

${ }^{2}$ Employs consecutive receipt of schema and instance(s)
}

tool functions that handle software representations are often deeply embedded and widely distributed throughout the code for the tool. A test that effectively checks incoming information must be based on all uses of the representation by the tool. In addition, the check must stay in sync with any modifications that are made over time to the exchange format or the internal representation within the tool. The effort involved in creating such a check in essence duplicates the efforts originally involved in handling the representation within the tool in the first place. As a consequence, it is unlikely that a tool that negotiates exchange using an implicit schema definition will include a check for well-formed input.

\subsection{Explicit Schema Definitions}

Exchange involving an explicit schema definition offers many benefits. The tool makes use of a specification or some other explicit means that identifies the structure and semantics of the information input. A clear separation exists between schema and instance data, no matter if the schema definition is internal or external. Because the definition is dynamic by nature, the representation is highly extensible. Modification of the representation is easily accomplished through changes made to the schema specification. All the information relating to the representation is located in a single location. This makes it easier for humans to get an overall understanding of the structure and semantics supported. The representation is always well documented and up to date. The explicit definition for the schema is itself the documentation.

Checking for well formed input is a straightforward process when the schema definition is explicit. The schema specification holds all the requirements that must be satisfied for the information to pass the checker. Implementing the checker is simple because the schema specification is complete and close at hand. The checker does not need to be maintained because the schema specification always outlines the current representation in use. For these reasons, it is likely that a tool that negotiates exchange using an explicit schema definition will include a check for wellformed input.

The main drawback of an explicit schema definition is 


\begin{tabular}{|c|c|c|c|}
\hline & & Advantages & Disadvantages \\
\hline \multirow{2}{*}{$\begin{array}{l}\text { Schema } \\
\text { Definition }\end{array}$} & Implicit & $\begin{array}{l}\text { - High performance no matter } \\
\text { how large the input }\end{array}$ & $\begin{array}{l}\text { - Not extensible } \\
\text { - Hard to document } \\
\text { - Difficult to implement check } \\
\text { for well formed input }\end{array}$ \\
\hline & Explicit & $\begin{array}{l}\text { - Highly Extensible } \\
\text { - Easily Understood } \\
\text { - Well documented } \\
\text { - Check for well formed input } \\
\text { is easier to implement }\end{array}$ & $\begin{array}{l}\text { - Low Performance } \\
\text { - Tool code is more complicated }\end{array}$ \\
\hline \multirow{2}{*}{$\begin{array}{l}\text { Schema } \\
\text { Locality }\end{array}$} & Internal & - High performance & $\begin{array}{l}\text { - Difficulties managing changes } \\
\text { among two or more tools }\end{array}$ \\
\hline & External & $\begin{array}{l}\text { - Easier to manage changes } \\
\text { among two or more tools }\end{array}$ & $\begin{array}{l}\text { Keeping the code consistent } \\
\text { with the schema is difficult }\end{array}$ \\
\hline
\end{tabular}

Table 2. Advantages and Disadvantages of Schema Definition and Locality on Exchange

that it requires interpretation. The schema must be processed before the tool can accept instance data. This intermediate step ultimately affects the performance of the tool. More importantly, there is a requirement for the tool to orient itself towards the representation provided in the schema. The tool must be flexible to accommodate this kind of functionality. In a best-case scenario, the tool would be able to accommodate any representation. In reality, it is likely that the representational capabilities of many tools will be limited. Building flexibility into a tool may also add significant complexity to the development effort.

\subsection{Internal Schemas}

The main advantage of an internal schema is accessibility. The tool does not need to venture out to an external source to determine the structure and semantics of the information model. This is an obvious advantage in terms of performance.

The difficulty with an internal schema definition becomes apparent when there is a need to change the information model. Maintaining conformity among two or more tools is difficult to achieve. This is especially challenging when the schema definition is implicit in all the affected tools. Changes must be implemented exhaustively throughout the code for each of the tools affected. Clearly an internal schema tends to make all tools participating in the exchange conform to a rigid representational structure and semantics.

\subsection{External Schemas}

With an external schema definition, managing conformity among two or more tools participating in the exchange is easily accomplished. A single schema definition is all that is necessary to ensure that each tool is using the correct structure and semantics for the representation being exchanged. Complete representational conformity among each tool participating in the exchange is assured as long as each tool makes use of the same schema definition. An external schema definition eliminates the need for a complete overhaul of the code for each tool when a change is made to the representation.

Nevertheless, the rules that each tool uses to process and analyze exchanged information can come out of sync with the schema because its definition is separated from the tool. Maintaining consistency between the code for a tool and the schema is challenging. The problem is exacerbated by the fact that external schemas are easily changed. The more often a schema is changed, the more likely that a loss of consistency will occur. The code in essence defines what the tool does with the information once it is successfully exchanged. But how this is accomplished is completely dependent on the structure and semantics of the representation defined externally by the schema.

Table 2 summarizes the relative advantages and disadvantages of each of the schema classifications.

\section{Exchange Pattern Satisfaction of SEF Re- quirements}

The requirements for a Standard Exchange Format (SEF) have been widely considered (for example, see [33, 36, 22, 27]). St-Denis et al. [32] list 13 requirements for an exchange format based on their past experiences and various requirements outlined in [3, 4, 6, 13, 23, 25, 28, 37].

In the following paragraphs we undertake an evaluation of exchange pattern alternatives with respect to the requirements of St-Denis et al. Visual indicators (defined in Ta- 
$\boldsymbol{x}$ The exchange pattern does not satisfy the requirement.

$\checkmark$ The exchange pattern satisfies the requirement.

$\checkmark \sqrt{ }$ The exchange pattern satisfies the requirement in a way that is particularly beneficial.

- The exchange pattern neither satisfies nor does not satisfy the requirement because it does not relate to the requirement.

\section{Table 3. Visual Indicators for SEF Require- ment Satisfaction}

ble 3) are used to provide an overall indication of how well the exchange pattern satisfies the requirement.

\subsection{Transparency}

Transparency of an exchange format is achieved when the use of encoders and decoders does not cause a loss, alteration or gain in the information being transferred. [32]

All Exchange Patterns (-) This requirement specifically deals with information handling procedures at both ends of the exchange process. None of the exchange patterns involve the use of encoders or decoders so transparency is not a related requirement.

\subsection{Scalability}

An exchange format is scalable when it is usable for exchanging information of all sizes, including representations of very large software applications. [32]

Implicit-Internal ( $\boldsymbol{X}$ ) Although an implicit schema definition provides high performance, the fact that the schema definition is embedded in the code means that the capacity of the tool is fixed. Making variations to the code to accommodate different magnitudes of information is difficult.

Explicit-Internal $(\boldsymbol{V})$ Although the explicit schema definition reduces the performance of the tool, it provides flexibility that makes it easier to adjust the representation to address scalability issues. For instance, one strategy for managing large bodies of information is to exchange only specific pieces of it rather than the whole thing. When the schema definition is explicit, adjusting the amount of information exchanged is much easier than when the definition is implicit.
Implicit-External (X) The implicit schema definition provides high performance but once again the tool is tailored to handle information in a particular way only. Although the schema might be easy to change because it is external, the tool may not be able to handle large volumes of information without significant code changes.

Explicit-External $(\boldsymbol{V})$ The advantages of this exchange pattern are identical to those for the explicit-internal exchange pattern, although the performance degradation may be more significant because the locality of the schema is external.

\subsection{Simplicity}

Simplicity is achieved when an exchange format is not complex or intricate. This makes it efficient, easier to describe, comprehend, apply and maintain while statistically reducing the prospects for errors and making it easier to process in an automated fashion. [32]

Implicit-Internal ( $\boldsymbol{x}$ ) The schema is indeed complex and intricate, being disseminated throughout the code for the tool. It is difficult to understand and maintain making it prone to erroneous modification.

Explicit-Internal $(\boldsymbol{})$ The non-embedded nature of the schema specification simplifies the exchange and makes it much easier to understand and maintain. The close proximity of the schema to the tool code provides greater efficiency over the explicit-external exchange pattern.

Implicit-External $(\boldsymbol{V} \checkmark)$ The schema does not exist which simplifies the exchange process and provides an environment where the throughput of information can be maximized.

Explicit-External $(\boldsymbol{V})$ The schema specification simplifies the exchange process, but its separation from the tool makes it less efficient than the explicit-internal exchange pattern.

\subsection{Neutrality}

Neutrality refers to an exchange format representation that is independent of any particular tool, so that as many tools as possible can integrate with it. [32]

Implicit-Internal ( $\boldsymbol{X})$ There is no neutrality of the representation as it is embedded into the code for the tool.

Explicit-Internal ( $\boldsymbol{x}$ ) The explicit nature of the schema definition provides a degree of neutrality. Nevertheless, the schema locality is internal to the tool, which 
impedes the integration of other tools to a standard representation.

Implicit-External ( $\boldsymbol{x}$ ) The schema is independent from the tool, which provides it with some degree of neutrality. Nevertheless, the schema is non-existent, so it is difficult to define a standard for other tools to integrate with it.

Explicit-External $(\boldsymbol{} \checkmark)$ Neutrality is maximized. The schema definition is completely separate from all tools and is explicitly defined. This makes it easier to integrate other tools to a standard representation.

\subsection{Formality}

Formal definition of an exchange format reduces the chances for misinterpretation and ensures that it is well understood by all parties. [32]

Implicit-Internal (x) There is no formal definition of the representation so it is very difficult to transfer knowledge of it to others. This is especially problematic because of the embedded nature of the representation.

Explicit-Internal $(\boldsymbol{V})$ By default, the explicit schema definition is formal. Because it is internal to the tool, all concerns relating to the tool implementation are together in the same place.

Implicit-External ( $\boldsymbol{x}$ ) The implicit nature of the representation means there is no formal definition. Because the schema locality is external, documentation must be relied upon for information on the representation.

Explicit-External $(\boldsymbol{V} \boldsymbol{})$ The explicit schema definition is itself a formal means for expressing the structure of the data instances. The external schema locality makes it easier for all tool integrators to understand the representation.

\subsection{Flexibility}

Flexibility is achieved when an exchange format accommodates different tools, languages and syntax for data and schemas. It also accommodates the exchange of incomplete information. [32]

Implicit-Internal ( $\boldsymbol{X})$ The exchange pattern offers no flexibility at all. The tool itself must handle any accommodation for different tools, languages or data/schema syntax.

Explicit-Internal $(\boldsymbol{})$ The explicit schema provides flexibility for changing the representation. This is partially negated by the fact that the schema is defined internally, which ties it very closely to the tool it is contained in.
Implicit-External ( $\boldsymbol{X}$ ) A certain degree of flexibility is offered by the implicit schema definition because it is external from all tools that participate in the exchange. Nevertheless, because the schema definition is implicit, it is difficult to offer representational flexibility. Each tool must conform to the same non-existent schema definition. This tends to force developers to keep to a rigid representational standard.

Explicit-External $(\boldsymbol{} \checkmark)$ Flexibility is maximized. First the schema definition is external, so it is not tied to any one tool. Second, the schema is explicitly defined so the representation is clear and easily modified.

\subsection{Evolvability}

An exchange format is evolvable when it can be changed easily to accommodate future needs. [32]

Implicit-Internal ( $\boldsymbol{X}$ ) Change is difficult to manage because the representation is embedded in the code for each tool.

Explicit-Internal (x) Although the explicit schema definition supports evolutionary changes, the internal locality of the schema ties the representation too closely with the tool. Changes to the representation must be implemented on a tool-by-tool basis.

Implicit-External ( $\boldsymbol{X})$ Although the schema definition is located externally, change is difficult to accommodate because the schema definition is implicit. Evolutionary changes are difficult to implement when all parties involved must approve it.

Explicit-External $(\boldsymbol{V} \boldsymbol{})$ Evolvability is maximized. The external schema definition does not tie the representation to any one tool. The explicit definition encourages evolutionary change in a collaborative manner.

\subsection{Popularity}

Popularity is achieved when the adoption of an exchange format is widespread so that as many tools as possible can take advantage of it. [32]

All Exchange Patterns (-) Exchange patterns that have external schemas may become more popular because they facilitate the use of well-accepted document exchange methods such as $X M L$. Nevertheless, the success of a particular integration technology ultimately rests with those who use it within the program comprehension community. 


\subsection{Completeness}

Completeness relates to the concept that everything needed to exchange information successfully is included. The user does not have to look after details relating to the exchange. [32]

All Exchange Patterns (-) We have differentiated between schema and instance data in the exchange process. Although these two components are required to carry out exchange (and in this way they typify how the exchange is managed), they do not represent a complete exchange format.

\subsection{Metamodel Identity}

This requirement refers to support for the transformation of instance data while preserving its identity. An integration technology preserves metamodel identity when it is capable of converting instance data from one schema into instance data of another. The instance data should remain the same; it is just represented differently from one schema to the next. [32]

Implicit-Internal $(\boldsymbol{X})$ The implicit nature of the schema definition makes it very difficult to support transformation of instance data. The use of the representation is embedded into the code for the tool. Identifying instance data and transforming it into an equivalent alternate representation is challenging.

Explicit-Internal $(\boldsymbol{V} \boldsymbol{})$ The schema definition is explicit which greatly assists in identifying the structure and semantics of instance data. At the same time, the schema is internal so it reflects the tool's view of instance data. Transformation of this schema to an external schema for exchange is all that is necessary.

Implicit-External ( $\boldsymbol{X}$ ) Once again, the implicit nature of the schema definition makes it very difficult to support transformation of instance data. The external schema definition is non-existent, which makes it difficult to identify a transformation to another schema that will preserve the identity of the instance data.

Explicit-External ( $\boldsymbol{V}$ ) The explicit schema definition lays out the representation in a single location external to the tool. Schema transformation can be carried out away from each of the tools participating in the exchange.

\subsection{Solution Reuse}

Solution reuse relates to the use of existing techniques and methods with the goal of reducing the amount of time and effort spent in testing and deploying an exchange format. [32]
Implicit-Internal $(\boldsymbol{x})$ The representation is embedded into the tool code, which makes it very difficult to reuse.

Explicit-Internal ( $\boldsymbol{X}$ ) Although explicitly defined, the representation remains closely tied to the tool. This tool centricity makes it difficult to reuse the representation outside the tool environment.

Implicit-External $(\boldsymbol{X})$ The non-existent schema definition is not easily described which makes it difficult to reuse.

Explicit-External $(\boldsymbol{V})$ The representation is defined explicitly and is not tied to any one tool. This tends to make it easier to reuse and makes it easier to implement and test.

\subsection{Legibility}

The legibility requirement relates to how easily a human reader can read and understand the format. [32]

Implicit-Internal ( $\boldsymbol{X})$ The embedded nature of the representation makes it difficult to understand, especially for non-programmers. Well-documented code may partially offset this problem.

Explicit-Internal $(\boldsymbol{V})$ Understanding of the representation is much easier when it is explicitly specified in a single location within the tool.

Implicit-External ( $\boldsymbol{X})$ The non-existent nature of the schema definition impedes understanding of the representation. This combined with the fact that the schema locality is external means that independent documentation must be relied upon to get information about the representation.

Explicit-External $(\boldsymbol{V})$ An explicit schema definition eases the legibility of the representation. The external locality of the schema ensures that the representation is tool independent.

\subsection{Integrity}

Integrity refers to the use of special mechanisms to ensure that information is exchanged without errors. [32]

All Exchange Patterns (-) The integrity of the exchange ultimately rests on the underlying technology used to communicate information.

\subsection{Comparative Summary}

Table 4 summarizes our evaluation of how each exchange pattern satisfies each SEF requirement. It is clear that the use of an explicit schema definition with external 


\begin{tabular}{|c|c|c|c|c|}
\hline \multirow[b]{2}{*}{ Requirements } & \multicolumn{4}{|c|}{ Exchange Pattern } \\
\hline & $\begin{array}{c}\text { Implicit- } \\
\text { Internal }\end{array}$ & $\begin{array}{l}\text { Explicit- } \\
\text { Internal }\end{array}$ & $\begin{array}{l}\text { Implicit- } \\
\text { External }\end{array}$ & $\begin{array}{l}\text { Explicit } \\
\text { External }\end{array}$ \\
\hline Transparency & - & - & - & - \\
\hline Scalability & $x$ & $\boldsymbol{\nu}$ & $x$ & $\boldsymbol{v}$ \\
\hline Simplicity & $x$ & $\checkmark$ & $\boldsymbol{V}$ & $\boldsymbol{v}$ \\
\hline Neutrality & $x$ & $x$ & $x$ & $\checkmark v$ \\
\hline Formality & $x$ & $\checkmark$ & $x$ & $\boldsymbol{V}$ \\
\hline Flexibility & $x$ & $\checkmark$ & $x$ & $\boldsymbol{V}$ \\
\hline Evolvability & $x$ & $x$ & $x$ & $\boldsymbol{V}$ \\
\hline Popularity & - & - & - & - \\
\hline Completeness & - & - & - & - \\
\hline Metamodel Identity & $x$ & $\boldsymbol{V}$ & $x$ & $\boldsymbol{V}$ \\
\hline Solution Reuse & $x$ & $x$ & $x$ & $\boldsymbol{v}$ \\
\hline Legibility & $x$ & $\boldsymbol{v}$ & $x$ & $\boldsymbol{v}$ \\
\hline Integrity & - & - & - & - \\
\hline
\end{tabular}

Table 4. Exchange Pattern Satisfaction of SEF Requirements

schema locality satisfies all the requirements that relate to exchange. In fact, five of the nine exchange-related requirements are strongly satisfied by the explicit-external exchange pattern. Following a distant second is the explicitinternal exchange pattern. Both of the exchange patterns with an implicit schema definition are the least satisfactory. Between these two, the implicit-external pattern is strongly beneficial solely because of its simplicity.

To summarize our evaluation, the use of schemas with an explicit definition and external locality are the preferred choice for an SEF. Explicit schema definition appears to be the most important factor in the evaluation.

\section{Conclusion}

Before tools can exchange information, they must share, at some level, the organization for the data exchanged. That is, they must share a schema. In this paper we examined the various ways in which schemas are represented and used in tools. Schema use was classified according to how and where a schema is defined, leading us to identify four patterns of exchange. We examined these exchange patterns and how each has been used in existing program comprehension systems. An evaluation of each exchange pattern against the requirements for a standard exchange format revealed that explicit-external schemas may be most suitable for integrating program comprehension tools.

Space limitations prevent us from detailing exactly how each of the requirements from St-Denis et al. [32] evaluated against each of the program comprehension tool integration technologies listed in Table 1. We hope to detail these in a future paper. Nevertheless, the observations in this paper strengthen the case for $G X L[15,16]$ as a standard exchange format. $G X L$ 's use of explicit-external schemas in combination with a metaschema for E-R graphs provides a common base from which any schema for representing software can be derived.

In this paper we have dealt with exchange issues at a relatively superficial level. In order to fully characterize the information exchange between tools, the issue of the schemas themselves must be addressed, but that is a topic for future work.

\section{References}

[1] ASIS Working Group. Ada Semantic Interface Specification. URL: http://www.acm.org/sigada/wg/ asiswg/.

[2] Project Bauhaus. URL: http://www. informatik. uni-stuttgart.de/ifi/ps/bauhaus/.

[3] I. T. Bowman, M. W. Godfrey, and R. C. Holt. "Connecting Architecture Reconstruction Frameworks". In Proceedings of the 1st International Symposium on Constructing Software Engineering Tools (CoSET'99), pages 43-54, Los Angeles, CA, May 1999.

[4] T. Bray. RDF and Metadata, June 1998. URL: http: // www. xml.com/pub/a/98/06/rdf.html.

[5] P. Chen. "The Entity Relationship Model - Toward a Unified View of Data". ACM Transactions on Database Systems, 1(1):9-36, 1976.

[6] R. Cover. The XML Cover Pages: XML and Semantic Transparency. Organization for the Advancement of Structured Information Standards (OASIS), November 1998. URL: http://www.oasis-open.org/ cover/xmlAndSemantics.html.

[7] J. Czeranski, T. Eisenbarth, H. M. Kienle, R. Koschke, E. Plödereder, D. Simon, Y. Zhang, J.-F. Girard, and 
M. Würthner. "Data Exchange in Bauhaus". In Proceedings of the Working Conference on Reverse Engineering (WCRE'00), Brisbane, Australia, November 2000.

[8] S. Demeyer, S. Ducasse, and S. Tichelaar. "Why FAMIX and not UML". In Proceedings of UML'99, volume 1723 of Lecture Notes in Computer Science. Springer-Verlag, 1999.

[9] J. Ebert, B. Kullbach, and A. Winter. "GraX - An Interchange Format for Reengineering Tools". In Proceedings of the 6th Working Conference on Reverse Engineering (WCRE'99), pages 89-98, 1999.

[10] J. Ebert, B. Kullbach, and A. Winter. "GraX: Graph Exchange Format". In Workshop on Standard Exchange Formats (WoSEF) at (ICSE'00), Limerick, Ireland, 2000.

[11] J. Ebert, A. Winter, P. Dahm, A. Franzke, and R. Süttenbach. "Graph Based Modeling and Implementation with EER/GRAL". In B. Thalheim, editor, Conceptual Modelling - ER'96, volume 1157 of Lecture Notes on Computer Science, pages 163-178, Berlin, 1996. Springer-Verlag.

[12] R. Ferenc, T. Gyimóthy, S. E. Sim, R. C. Holt, and R. Koschke. "Towards a Standard Schema for $\mathrm{C} / \mathrm{C}++$ ". In Proceedings of the 8th Working Conference on Reverse Engineering (WCRE'01), Stuttgart, Germany, October 2001.

[13] R. Holt. An Introduction to TA: The Tuple Attribute Language. Department of Computer Science, University of Waterloo and University of Toronto, November 1998.

[14] R. C. Holt. "Structural Manipulations of Software Architecture Using Tarski Relational Algebra". In Proceedings of the 5th Working Conference on Reverse Engineering (WCRE'98), Honolulu, Hawaii, October 1998.

[15] R. C. Holt and A. Winter. "A Short Introduction to the GXL Exchange Format". In Proceedings of the 7th Working Conference on Reverse Engineering (WCRE'00), Panel on Reengineering Exchange Formats, 2000.

[16] R. C. Holt and A. Winter. GXL: Representing Graph Schemas. Presented at the 7th Working Conference on Reverse Engineering (WCRE'00), 2000.

[17] International Organization for Standardization. Ada Semantic Interface Specification (ASIS), Edition 1. 283 pages, Stage Date: April 29, 1999.

[18] D. Jin. "Exchange Of Software Representations Among Reverse Engineering Tools". Technical Report 2001-454, Department of Computing and Information Science, Queen's University, Kingston, Canada, December 2001.

[19] R. Kazman, S. G. Woods, and S. J. Carrière. "Requirements for Integrating Software Architecture and Reengineering Models: CORUM II". In Proceedings of the Working Conference on Reverse Engineering (WCRE'98), October 1998 .

[20] S. Lapierre. Software Analysis APIs. Presented at the ICSE 2000 Workshop on Standard Exchange Formats (WoSEF), June 6, 2000

[21] S. Lapierre, B. Laguë, and C. Leduc. "Datrix Source Code Model and its Interchange Format: Lessons Learned and Considerations for Future Work". Software Engineering Notes, 26(1):53-56, January 2001.

[22] T. C. Lethbridge. Requirements and Proposal for a Software Information Exchange Format (SIEF) Standard, November 1998. URL: http://www.site.uottawa.ca/ tcl/papers / sief/standardProposalvl.html.
[23] T. C. Lethbridge and N. Anquetil. "Architecture of a Source Code Exploration Tool: A Software Engineering Case Study". Technical Report TR-97-07, School of Information Technology and Engineering (SITE), University of Ottawa, November 1997.

[24] T. C. Lethbridge, E. Plödereder, S. Tichelaar, C. Riva, and P. Linos. The Dagstuhl Middle Model (DMM). Version 0.003, June 6, 2001.

[25] H. A. Müller. Criteria for Success of an Exchange Format. Workshop meeting minutes, CASCON'98, Nov. 30, 1998.

[26] H. A. Müller and K. Klashinsky. "Rigi - A system for Programming-in-the-Large". In Proceedings of the International Conference on Software Engineering (ICSE'88), pages 80-86, 1988.

[27] H. A. Müller, K. Wong, and S. R. Tilley. "Understanding Software Systems Using Reverse Engineering Technology". In Proceedings of the 62nd Congress of L'Association Canadienne Francaise pour l'Avancement des Sciences (ACFAS), 1994.

[28] Object Management Group. XML Metadata Interchange (XMI), Proposal to the OMG OA\&DTF RFP 3: Streambased Model Interchange Format (SMIF). OMG Document ad/98-10-05, October 20, 1998.

[29] A. Schürr. "Developing Graphical (Software Engineering) Tools with PROGRES". In Proceedings of the 19th International Conference on Software Engineering (ICSE'97), pages 618-619, Boston, Massachusetts, May 1997.

[30] A. Schürr. PROGRES for Beginners. Department of Computer Science, Aachen University of Technology, 1997.

[31] S. E. Sim, R. C. Holt, and R. Koschke. WoSEF - Workgroup on Standard Exchange Format, Progress Towards a Format. URL: http://www.cs.toronto.edu/ simsuz/wosef/.

[32] G. St-Denis, R. Schauer, and R. K. Keller. "Selecting a Model Interchange Format: The SPOOL Case Study". In Proceedings of the 33rd Annual Hawaii International Conference on System Sciences, Maui, Hawaii, January 2000.

[33] S. Tichelaar, S. Ducasse, and S. Demeyer. "FAMIX: Exchange Experiences with CDIF and XMI". In Workshop on Standard Exchange Formats (WoSEF) at (ICSE'O0), Limerick, Ireland, 2000.

[34] Introduction to TkSee 2.0. URL: http://www. site. uottawa.ca/ tcl/kbre/options/intro.html.

[35] M. van den Brand, H. de Jong, P. Klint, and P. Olivier. "Efficient Annotated Terms". Software, Practice \& Experience, 30:259-291, 2000.

[36] M. van den Brand, H. de Jong, and P. Olivier. "A Common Exchange Format for Reeingineering Tools Based on ATerms". In Workshop on Standard Exchange Formats (WoSEF) at (ICSE'O0), Limerick, Ireland, 2000.

[37] S. Woods, S. J. Carrière, and R. Kazman. "A Semantic Foundation for Architectural Reengineering and Interchange". In Proceeding of the 1999 International Conference on Software Maintenance (ICSM'99), pages 391-398, Oxford, UK, August 1999.

[38] S. Woods, L. O’Brien, T. Lin, K. Gallagher, and A. Quilici. "An Architecture For Interoperable Program Understanding Tools". In Proceeding of the 6th International Workshop on Program Comprehension (IWPC'98), pages 54-63, 1998. 